\title{
Theoretical Framework for Constructing Matching Algorithms in Biometric Authentication Systems
}

\author{
Manabu Inuma ${ }^{1,2}$, Akira Otsuka ${ }^{1,2}$, and Hideki Imai ${ }^{1,2}$ \\ 1 Research Center for Information Security (RCIS) \\ National Institute of Advanced Industrial Science and Technology (AIST) \\ Akihabara-Daibiru Room 1003, 1-18-13, Sotokanda, Chiyoda-ku \\ Tokyo 101-0021, Japan \\ 2 Department of Electrical, Electronic, and Communication Engineering \\ Faculty of Science and Engineering, Chuo University \\ 1-13-27 Kasuga, Bunkyo-ku Tokyo 112-8551, Japan
}

\begin{abstract}
In this paper, we propose a theoretical framework to construct matching algorithms for any biometric authentication systems. Conventional matching algorithms are not necessarily secure against strong intentional impersonation attacks such as wolf attacks. The wolf attack is an attempt to impersonate a genuine user by presenting a "wolf" to a biometric authentication system without the knowledge of a genuine user's biometric sample. A "wolf" is a sample which can be accepted as a match with multiple templates. The wolf attack probability (WAP) is the maximum success probability of the wolf attack, which was proposed by Une, Otsuka, Imai as a measure for evaluating security of biometric authentication systems [UI1, [UOI2]. We present a principle for construction of secure matching algorithms against the wolf attack for any biometric authentication systems. The ideal matching algorithm determines a threshold for each input value depending on the entropy of the probability distribution of the (Hamming) distances. Then we show that if the information about the probability distribution for each input value is perfectly given, then our matching algorithm is secure against the wolf attack. Our generalized matching algorithm gives a theoretical framework to construct secure matching algorithms. How lower WAP is achievable depends on how accurately the entropy is estimated. Then there is a trade-off between the efficiency and the achievable WAP. Almost every conventional matching algorithm employs a fixed threshold and hence it can be regarded as an efficient but insecure instance of our theoretical framework. Daugman's algorithm proposed in [Da2] can also be regarded as a non-optimal instance of our framework.
\end{abstract}

\section{Introduction}

Biometric authentication systems automatically identify or verify individuals by physiological or behavioral characteristics. They are used in various services such as the immigration control at an airport, the banking transactions at an ATM,

M. Tistarelli and M.S. Nixon (Eds.): ICB 2009, LNCS 5558, pp. 806-815 2009.

(C) Springer-Verlag Berlin Heidelberg 2009 
the access control to restricted areas in a building, and so on. The increase in the need of biometric authentication systems makes it important to explicitly evaluate the security of them.

The false acceptance rate $(F A R)$ (see the definition (3) in Section 2.2) is traditionally used as a security measure against the zero-effort impersonation attack. The zero-effort approach assumes that an attacker will present his/her own biometric data. But, it is clearly not a rational assumption, since an attacker attempting to impersonate a genuine user will try to present a biometric data of the genuine user or its imitation.

Ratha et al. approximately calculate the success probability of a brute-force attack in a typical fingerprint-minutiae matching algorithm RCB1. The bruteforce approach assumes that an attacker blindly selects an input value. However, if an attacker has some information about the algorithm employed in the system, the attacker might be able to find a sample which shows high similarity to most of the templates. Such a biometric sample is called a wolf (cf. [ISO1]). An attacker could impersonate a genuine user with much higher probability than $F A R$ by presenting a wolf to a biometric authentication system.

With regard to the artefact attack, Matsumoto et al. showed that some biometric authentication systems often falsely accepts some artefacts [MMYH1. Therefore we should assume that an attacker may find a special input value not only from biometric samples but also from non-biometric samples. Une, Otsuka, Imai extended the definition of a wolf to include a non-biometric input value and defined the wolf attack probability ( $W A P$ ) (see Definition 3) [UOI], UOI2]. $W A P$ can be regarded as the upper bound of the success probability of attacks without the knowledge of a genuine user's biometric sample. Une, Otsuka, Imai proposed that $W A P$ can be used as a security measure to evaluate the lower bound of a security level in a biometric authentication system.

Our goal is to propose a theoretical framework to construct matching algorithms for biometric authentication systems. Almost every conventional matching algorithm employs a fixed threshold determined based on FAR and the false rejection rate $(F R R)$ (see the definition (1) in Section 2.1). It is not necessarily secure against the wolf attack. Une, Otsuka, Imai showed that in some of such matching algorithms, there actually exist strong wolves and $W A P$ can be extremely higher [UOI1], [UOI2]. Surprisingly, as far as we know, no research have been conducted on security of matching algorithms until now. This is the first paper which studies the security characteristics of matching algorithms and gives a theoretical framework how to construct them securely.

Suppose a matching algorithm employs a threshold determined by the entropy of the probability distribution for each input value. We prove that if the entropy for each input value is perfectly given, then the matching algorithm is secure against the wolf attack (Theorem 1, 2).

In the real world, it might be difficult to perfectly calculate the entropy for each input value, however, a more accurate computation of the entropy can achieve a lower $W A P$. Then there is a trade-off between the efficiency of the matching algorithm and the achievable $W A P$ in the matching algorithm. 
Previous results can be regarded as instances of our theoretical framework. Almost every previous matching algorithm employs a fixed threshold. In our theoretical framework, it can be regarded as an efficient instance which assumes all input values have the same entropy. However, as mentioned above, it is not exactly secure against the wolf attack.

Daugman proposed a matching algorithm in which a threshold is determined for each match by taking account the number of bits available for comparison Da2. His method can also be regarded as an instance of our framework, which assumes every bit of a sample independently and identically contributes to the entropy of the probability distribution. WAP in his algorithm can be relatively lower than that in an ordinary algorithm employing a fixed threshold. However, his matching algorithm is not necessarily secure against the wolf attack (see details in Section (4), since we have to assume that an attacker knows more accurate information about the probability distributions.

This paper continues as follows. In Section 2 , we will briefly introduce a typical model of biometric authentication systems and give explicit definitions of $F A R$, $W A P$, and security against the wolf attack. Our proposal can be easily adapted to all matching algorithms of all modalities that employ symmetric prametric functions such as the ordinary (Hamming) distance as the dissimilarity measure. We will construct matching algorithms in the general case (Theorem 1) and in the normal distribution case (Theorem 2) and show that these matching algorithms are secure against the wolf attack. They give a theoretical framework to construct secure matching algorithms for any biometric authentication systems. In Section 4, we will reconsider previous results in our theoretical framework.

\section{Model (Preliminaries)}

A biometric authentication system can be used for verification or identification of individuals. In verification, a user of the system claims to have a certain identity and the biometric system performs a one to one comparison between the offered biometric data and the template which is linked to the claimed identity. In identification, a one to all comparison is performed between the offered data and all available template stored in the database to reveal the identity of an individual. In this paper, we will discuss verification systems.

Let $\mathcal{U}$ be a set of all possible users of the biometric authentication system. Namely $\mathcal{U}$ is a set of all human individuals. For each user $u \in \mathcal{U}$, the identity of $u$ can be denoted by $u$, namely the identities of users can be identified with $\mathcal{U}$. Let $\mathcal{M}$ be a finite set with a symmetric prametric function $d: \mathcal{M} \times \mathcal{M} \rightarrow \mathbb{R}$, namely $d(x, y)=d(y, x), d(x, y) \geq 0, d(x, x)=0$ for all $x, y \in \mathcal{M}$.

In an enrollment phase, for any user $u \in \mathcal{U}$, an acquisition device measures a biometric data of $u$. After processing the measurement data and extracting relevant features, the features are represented as an element $t_{u}$ of $\mathcal{M}$. Then the template $t_{u}$ of $u \in \mathcal{U}$ is stored in the database of the system. In a verification phase (matching phase) match, a user $v \in \mathcal{U}$ claims an identity $w \in \mathcal{U}$ and a biometric measurement is acquired from $v$. This measurement is also transformed 
into an element $s$ of $\mathcal{M}$. A matching process compares $s$ with $t_{w}$ and match generates a message, accept or reject, by a predetermined threshold $\tau \in \mathbb{R}_{\geq 0}$ as follows:

$$
\operatorname{match}(v, w)=\left\{\begin{array}{l}
\text { accept if } d\left(s, t_{w}\right)<\tau \\
\text { reject if } d\left(s, t_{w}\right) \geq \tau
\end{array} .\right.
$$

Each user $u \in \mathcal{U}$ enrolls and offers a certain biometric sample of $u$ in an enrollment phase and a verification phase, respectively. Therefore $\mathcal{U}$ can be regarded as a set of the biometric samples of users. For each biometric sample $u \in \mathcal{U}$, let $X_{u}$ be a random variable on $\mathcal{M}$ representing noisy versions of $u$, namely $P\left(X_{u}=s\right)$ denotes the probability that biometric data of $u$ will be transformed into $s \in \mathcal{M}$. Assume that the $X_{u}, u \in \mathcal{U}$, are independent.

\subsection{The False Rejection Rate}

The false rejection rate $(F R R)$ is the probability that a genuine user is rejected, namely it is defined by

$$
\begin{aligned}
F R R & =\underset{u \in \mathcal{U}}{\operatorname{Ave}} P(\operatorname{match}(u, u)=\text { reject }) \\
& =\frac{1}{n} \sum_{u \in \mathcal{U}} \sum_{\substack{(s, t) \in \mathcal{M} \times \mathcal{M} \\
d(s, t) \geq \tau}} P\left(X_{u}=s\right) P\left(X_{u}=t\right) \\
& =1-\frac{1}{n} \sum_{u \in \mathcal{U}} \sum_{\substack{(s, t) \in \mathcal{M} \times \mathcal{M} \\
d(s, t)<\tau}} P\left(X_{u}=s\right) P\left(X_{u}=t\right)
\end{aligned}
$$

where $n=\# \mathcal{U}$. For each user $u \in \mathcal{U}$, let $F R R_{u}$ denote the probability that the user $u$ with the correct identity claim $u$ will be rejected. Namely, $F R R_{u}$ is defined by

$$
\begin{aligned}
F R R_{u} & =\sum_{\substack{(s, t) \in \mathcal{M} \times \mathcal{M} \\
d(s, t) \geq \tau}} P\left(X_{u}=s\right) P\left(X_{v}=t\right) \\
& =1-\sum_{\substack{(s, t) \in \mathcal{M} \times \mathcal{M} \\
d(s, t)<\tau}} P\left(X_{u}=s\right) P\left(X_{v}=t\right)
\end{aligned}
$$

It is easy to check that $F R R=\frac{1}{n} \sum_{u \in \mathcal{U}} F R R_{u}$.

\subsection{The False Acceptance Rate}

The false acceptance rate $(F A R)$ is the probability that an offer of a user with a wrong identity claim will be incorrectly accepted, namely $F A R$ is defined by

$$
\begin{aligned}
F A R & =\underset{\substack{(u, v) \in \mathcal{U} \times \mathcal{U} \\
u \neq v}}{\operatorname{Ave}} P(\operatorname{match}(u, v)=\text { accept }) \\
& =\frac{1}{n(n-1)} \sum_{\substack{(u, v) \in \mathcal{U} \times \mathcal{U} \\
u \neq v}} \sum_{\substack{(s, t) \in \mathcal{M} \times \mathcal{M} \\
d(s, t)<\tau}} P\left(X_{u}=s\right) P\left(X_{v}=t\right) .
\end{aligned}
$$


The measure $F A R$ is traditionally used to express a recognition accuracy of biometric systems. It is also used as a measure to evaluate the security of systems against the zero-effort impersonation attack.

The zero-effort approach assumes that an attacker attempting to impersonate a genuine user will present his/her own biometric data. This assumption is clearly so far from reality, since an attacker will try to present a biometric data of a genuine user or its imitation.

\subsection{The Wolf Attack Probability}

Une, Otsuka, Imai proposed a new security measure for biometric authentication systems [UOI1, UOI2. If an attacker can find an input value which matches many templates, then he succeed in impersonating a genuine user with a higher probability than $F A R$ by presenting the input value to the biometric authentication system. Such an input value obtained from a biometric sample is called a wolf by many authors (cf. [ISO1]). However, such an input value might be obtained not only from biometric samples but also from non-biometric samples. Matsumoto et al. show by experimentation that some artefacts can be falsely accepted in some biometric authentication systems [MMYH1.

Considering these facts, we will extend the definition of a wolf as follows.

Let $\mathcal{A}$ be a set of all possible samples including non-biometric samples such as artefacts or synthetic samples. For each $w \in \mathcal{A}$, let $F A R_{w}$ denote the probability that the sample $w$ with a wrong identity claim $v \neq w$ will be incorrectly accepted and let $A R_{w}$ denote the probability that the sample $w$ with random claim will be accepted. Namely, $F A R_{w}$ and $A R_{w}$ are respectively defined by

$$
\begin{aligned}
F A R_{w} & =\underset{\substack{v \in \mathcal{U} \backslash\{w\} \\
\text { Ave }}}{ } P(\text { match }(w, v)=\text { accept }) \\
& =\frac{1}{\#(\mathcal{U} \backslash\{w\})} \sum_{v \in \mathcal{U} \backslash\{w\}} \sum_{\substack{(s, t) \in \mathcal{M} \times \mathcal{M} \\
d(s, t)<\tau}} P\left(X_{w}=s\right) P\left(X_{v}=t\right), \\
A R_{w} & =\operatorname{Ave}_{v \in \mathcal{U}} P(\operatorname{match}(w, v)=\text { accept }) \\
& =\frac{1}{n} \sum_{v \in \mathcal{U}} \sum_{\substack{(s, t) \in \mathcal{M} \times \mathcal{M} \\
d(s, t)<\tau}} P\left(X_{w}=s\right) P\left(X_{v}=t\right) .
\end{aligned}
$$

It is easy to check that $F A R=\frac{1}{n} \sum_{u \in \mathcal{U}} F A R_{u}$. The following theorem describes the relation between $F R R_{w}, F A R_{w}$ and $A R_{w}$.

\section{Lemma 1}

$$
A R_{w}=\left\{\begin{array}{lr}
F A R_{w} & \text { if } w \in \mathcal{A} \backslash \mathcal{U} \\
\frac{1}{n}\left(1-F R R_{w}\right)+\left(1-\frac{1}{n}\right) F A R_{w} & \text { if } w \in \mathcal{U}
\end{array}\right.
$$


Therefore it immediately follows that

$$
\frac{1}{n} \sum_{u \in \mathcal{U}} A R_{u}=\frac{1}{n}(1-F R R)+\left(1-\frac{1}{n}\right) F A R .
$$

Proof. For any $w \in \mathcal{A} \backslash \mathcal{U}$, it is clear that $A R_{w}=F A R_{w}$, since $\mathcal{U} \backslash\{w\}=\mathcal{U}$. For any $w \in \mathcal{U}$, from the definitions (2), (4) and (5) of $F R R_{w}, F A R_{w}$ and $A R_{w}$, respectively, we have

$$
\begin{aligned}
A R_{w}= & \frac{1}{n} \sum_{\substack{(s, t) \in \mathcal{M} \times \mathcal{M} \\
d(s, t)<\tau}} P\left(X_{w}=s\right) P\left(X_{w}=t\right) \\
& +\frac{n-1}{n} \cdot \frac{1}{n-1} \sum_{v \in \mathcal{U} \backslash\{w\}} \sum_{\substack{(s, t) \in \mathcal{M} \times \mathcal{M} \\
d(s, t)<\tau}} P\left(X_{w}=s\right) P\left(X_{v}=t\right) \\
= & \frac{1}{n}\left(1-F R R_{w}\right)+\left(1-\frac{1}{n}\right) F A R_{w} .
\end{aligned}
$$

Therefore the results follow.

Put

$$
A R=\frac{1}{n} \sum_{u \in \mathcal{U}} A R_{u}=\frac{1}{n}(1-F R R)+\left(1-\frac{1}{n}\right) F A R .
$$

Note that usual biometric authentication systems obviously satisfy $F A R \leq 1-$ $F R R$, namely $F A R \leq A R$.

Definition 1. (cf. UOI1, Definition 3]) $A$ wolf is defined as a sample $w \in \mathcal{A}$ such that $A R_{w}>A R$.

For any $A R<p \leq 1$, a wolf $w$ such that $A R_{w}=p$ is called a $p$-wolf. In particular, 1-wolf is called a universal wolf.

Definition 2. UOI1, Definition 4] Assume the following two conditions.

(i) The attacker has no information of a biometric sample of a genuine user to be impersonated.

(ii) The attacker has complete information of the algorithms employed in the enrollment phase and the verification phase.

The wolf attack is defined as an attempt to impersonate a genuine user by presenting p-wolves with large p's to minimize the complexity of the impersonation attack.

Definition 3 (Wolf attack probability (WAP)). (cf. [UOI1, Definition 5]) The Wolf attack probability is defined by

$$
W A P=\max _{w \in \mathcal{A}} \operatorname{Ave}_{v \in \mathcal{U}} P(\operatorname{match}(w, v)=\text { accept })=\max _{w \in \mathcal{A}} A R_{w}
$$


It is clear that $A R \leq W A P$. Therefore, if $F A R \leq 1-F R R$, then we have $F A R \leq A R=\underset{u \in \mathcal{U}}{\operatorname{Ave}} A R_{u} \leq W A P$.

Definition 4 (Security against the wolf attack). For any $\delta>0$, a biometric authentication system is $\delta$-secure against the wolf attack if $W A P<\delta$, namely there exist no wolf $w \in \mathcal{A}$ such that $A R_{w} \geq \delta$.

If we use only FAR as a security measure against impersonation attacks, then we cannot explicitly evaluate the security against wolf attacks. Une, Otsuka, Imai proposed to evaluate the security level against the wolf attack by computing $W A P$ UOI1, UOI2.

\section{Matching Algorithms Secure against the Wolf Attack}

For each $s \in \mathcal{M}$ and $x \in \mathbb{R}_{\geq 0}$, the probability $P_{s}(x)$ that a template $t \in \mathcal{M}$ obtained from a biometric sample of a user will be at a distance less than $x$ from $s$ is defined by

$$
P_{s}(x)=\frac{1}{n} \sum_{v \in \mathcal{U}} \sum_{\substack{t \in \mathcal{M} \\ d(s, t)<x}} P\left(X_{v}=t\right)
$$

Then we have

$$
A R_{w}=\sum_{s \in \mathcal{M}} P\left(X_{w}=s\right) P_{s}(\tau)
$$

\subsection{General Case}

Fix $\delta>0$. Then we will construct a matching algorithm $\delta$-secure against the wolf attack as follows.

Almost every conventional matching algorithm employs a fixed threshold $\tau$ predetermined based on FRR and FAR. However, we will employ a threshold $\tau_{s}$ determined for each element $s \in \mathcal{M}$ obtained from the sample $w \in \mathcal{A}$ offered in the verification phase. For each $s \in \mathcal{M}$, put

$$
\tau_{s}=\max \left\{x \in \mathbb{R}_{\geq 0} \mid P_{s}(x)<\delta\right\} .
$$

Note that a set $S=\left\{x \in \mathbb{R}_{\geq 0} \mid P_{s}(x)<\delta\right\}$ is a non-empty closed subset of $\mathbb{R}_{\geq 0}$ and therefore there exists the maximum of $S$.

For the implementation, we need to gather enough templates from each $v \in \mathcal{U}$ and estimate the probabilities $P\left(X_{v}=t\right)$ for all $t \in \mathcal{M}$. Then we can determine the threshold $\tau_{s}$ for each $s \in \mathcal{M}$ by doing the exhaustive search of all possible $x \geq 0$ such that $P_{s}(x)<\delta$.

It is clear that

$$
W A P=\max _{w \in \mathcal{A}} \sum_{s \in \mathcal{M}} P\left(X_{w}=s\right) P_{s}\left(\tau_{s}\right)<\delta .
$$

The above discussion gives the following theorem. 
Theorem 1. If the information about the probability distribution $P_{s}(x)$ for each $s \in \mathcal{M}$ is completely given, then, for any $\delta>0$, we can construct a matching algorithm $\delta$-secure against the wolf attack.

\subsection{Normal Distribution Case}

We assume that the distribution $P_{s}(x)$ is normal with mean $m_{s}$ and standard deviation $\sigma_{s}$ for each $s \in \mathcal{M}$, namely

$$
P_{s}(x)=\int_{-\infty}^{x} \frac{1}{\sqrt{2 \pi} \sigma_{s}} \exp \left(-\frac{1}{2}\left(\frac{x-m_{s}}{\sigma_{s}}\right)^{2}\right) d x
$$

for any $x>0$. More strictly, we assume that $P_{s}(x)$ can be approximately estimated by the above equation. The distributions of Hamming distances for Daugman's iriscode satisfy this assumption (cf. Da1, Da2 ). Some authors use the Gaussian assumption as the basis of their analysis (cf. AYL1, Ka1, Wa1]). In general, the real-valued features will tend to approximate a Gaussian distribution when they are obtained by a linear combinations of many components, e.g. feature extraction techniques based on the principle component analysis (PCA) or the linear discriminant analysis (LDA) (cf. [AYL1]). Under this assumption, we can construct a secure and simple matching algorithm and show that the matching algorithm is optimal, namely $W A P$ is minimized to the (almost) same value as $A R$.

Define the entropy $H(P)$ of the probability distribution $P$ by

$$
H(P)=\int_{-\infty}^{\infty}-P(x) \log _{2} P(x) d x .
$$

By the assumption (10), it can be easily checked that

$$
H\left(P_{s}\right)=\log _{2}\left(\sqrt{2 \pi e} \cdot \sigma_{s}\right) .
$$

We work with entropies $H(P)$ of continuous probability distributions $P$. Then the entropy $H(P)$ is not always non-negative. It is clear from (11) that if $\sigma_{s}<$ $\frac{1}{\sqrt{2 \pi e}}$, then $H\left(P_{s}\right)<0$. Note that if a fixed threshold is employed, then an input value $s \in \mathcal{M}$ which has higher entropy $H\left(P_{s}\right)$ and therefore larger deviation $\sigma_{s}$ can be accepted with higher probability.

Fix a real number $\alpha$. For each $s \in \mathcal{M}$, put

$$
\tau_{s}=\alpha \sigma_{s}+m_{s}=\frac{\alpha 2^{H_{s}}}{\sqrt{2 \pi e}}+m_{s}
$$

where $H_{s}=H\left(P_{s}\right)$. By the assumption (10), we have

$$
P_{s}\left(\tau_{s}\right)=\int_{-\infty}^{\tau_{s}} \frac{1}{\sqrt{2 \pi} \sigma_{s}} \exp \left(-\frac{1}{2}\left(\frac{x-m_{s}}{\sigma_{s}}\right)^{2}\right) d x=\int_{-\infty}^{\alpha} \frac{1}{\sqrt{2 \pi}} \exp \left(-\frac{z^{2}}{2}\right) d z
$$


for all $s \in \mathcal{M}$. Put

$$
\delta(\alpha)=\int_{-\infty}^{\alpha} \frac{1}{\sqrt{2 \pi}} \exp \left(-\frac{z^{2}}{2}\right) d z \quad .
$$

The following theorem can be immediately proved.

Theorem 2. Assume that the standard deviation $\sigma_{s}$ (or the entropy $H_{s}$ ) and the mean $m_{s}$ are perfectly given for each $s \in \mathcal{M}$. Then the matching algorithm employing the thresholds $\tau_{s}, s \in \mathcal{M}$, defined by (12) is $\delta(\alpha)$-secure against wolf attacks. Moreover, we have $A R_{w}=A R=W A P=\delta(\alpha)$ for all $w \in \mathcal{A}$.

Proof. By the calculation (13), for all $w \in \mathcal{A}$, we have

$$
A R_{w}=\sum_{s \in \mathcal{M}} P\left(X_{w}=s\right) P_{s}\left(\tau_{s}\right)=\delta(\alpha) .
$$

Therefore the results follow.

Our generalized matching algorithm gives a theoretical framework for constructing matching algorithms secure against the wolf attack for any biometric authentication system. Under the ideal condition that for each $s \in \mathcal{M}$, the distribution $P_{s}(x)$ is completely calculated, our matching algorithm is optimal against the wolf attack.

In the real world, it might be difficult to explicitly calculate the distribution $P_{s}(x)$ for all $s \in \mathcal{M}$, however, a more accurate computation of $\sigma_{s}, H_{s}$, or $m_{s}$ for each $s \in \mathcal{M}$ can achieve a lower $W A P$. Consequently, there is a trade-off between the efficiency of the matching algorithm and the security evaluated by the achievable $W A P$. In the next section, we will reconsider previous results as instances of our theoretical framework.

\section{Previous Results in Our Framework}

In this section, we will review previous results in the context of our theoretical framework.

A conventional matching algorithm employing a fixed threshold can be viewed as an efficient instance of our framework, which assumes every input value has a constant entropy instead of computing the entropy for each input value. Such a matching algorithm is not secure against the wolf attack.

Daugman proposes a matching algorithm which employs a variable threshold in place of a fixed threshold as follows Da2. He employs a fractional Hamming distance $d=f H D$ defined by $f H D(s, t)=\frac{H D(s, t)}{k}$ for any $s, t \in \mathcal{M}=\{0,1\}^{2048}$, where $k$ is the number of bits available for comparison. He determines a threshold depending on $k$ as follows:

$$
\tau(s, t)=\frac{\alpha^{\prime}}{\sqrt{k}}+\frac{1}{2}
$$


where $\frac{1}{2}$ is the average of $f H D(s, t)$ estimated from his database. His algorithm can also be regarded as an instance of our framework, which assumes every bit of each sample independently and identically contributes to the probability distribution.

However, his algorithm is not necessarily secure against the wolf attack, since every bit is not exactly independent and identical and the distributions $P_{s}(x), s \in$ $\mathcal{M}$, can be considerably different from each other. We assume that an attacker has more accurate information about the distributions $P_{s}(x), s \in \mathcal{M}$. If the attacker can successfully find a smart input value $s \in \mathcal{M}$ such that the entropy $H\left(P_{s}(x)\right)$ is extremely high, then he can be incorrectly accepted with much higher probability than $A R$.

Daugman's matching algorithm is not always secure against the wolf attack, however, it motivated us to research a theoretical framework to construct secure matching algorithms.

\section{References}

[AYL1] Adler, A., Youmaran, R., Loyka, S.: Towards a measure of biometric feature information. In: Pattern Analysis and Applications. Springer, London (Online first), doi:10.1007/s10044-008-0120-3

[Da1] Daugman, J.: High Confidence Visual Recognition of Persons by a Test of Statstical Independence. IEEE Trans. on Pattern Anal. Mach. Intell. 15(11) (November 1993)

[Da2] Daugman, J.: Probing the uniqueness and randomness of IrisCodes: Results from 200 billion iris pair comparisons. Proceedings of the IEEE 94(11), 1927-1935 (2006)

[ISO1] International Organization for Standardization (ISO), International Electronical Commission (ICE): ISO/IEC CD 19792: Imformation technology - Security techniques - Security evaluations of biometrics (2006)

[Ka1] Kevenaar, T.: Protection of Biometric Information. In: Tuyls, P., Skoric, B., Kevenaar, T. (eds.) Security with Noisy Data: On Private Biometrics, Secure Key Storage and Anti-Counterfeiting, ch. 7, pp. 113-125. Springer, London (2007)

[MMYH1] Matsumoto, T., Matsumoto, H., Yamada, K., Hoshino, S.: Impact of Artificial 'Gummy' Fingers on Fingerprinting Systems. In: Opt. Sec. and Count. Det. Tech. IV, Proc. of SPIE, vol. 4677, pp. 275-289 (2002)

[RCB1] Ratha, N.K., Connell, J.H., Bolle, R.M.: Enhancing security and privacy in biometrics-based authentication systems. IBM Syst. J. 40, 614-634 (2001)

[UOI1] Une, M., Otsuka, A., Imai, H.: Wolf Attack Probability: A New Security Measure in Biometric Authentication Systems. In: Lee, S.-W., Li, S.Z. (eds.) ICB 2007. LNCS, vol. 4642, pp. 396-406. Springer, Heidelberg (2007)

[UOI2] Une, M., Otsuka, A., Imai, H.: Wolf Attack Probability: A Theoretical Security Measure in Biometrics-Based Authentication Systems. IEICE, Transactions on Information and Systems 2008 E91-D (5), 1380-1389 (2008)

[Wa1] Wayman, J.S.: The cotton ball problem. In: Biometrics Conference, Washington, DC, USA, September 20-22 (2004) 